\title{
Pembrolizumab-Induced Hepatitis and Pancreatitis in a Patient With Stage IV Non-Small Cell Lung Cancer
}

\author{
Rajveer Joea ${ }^{a}$, Muhammad Muftia, d, Jennifer Oberharta, \\ Sarkissian Sarmen ${ }^{\mathrm{b}, \mathrm{c}}$
}

\begin{abstract}
Immune checkpoint inhibitors are a novel approach to treat cancers. Firstly used for treatment of malignant melanomas with promising results, they were later expanded to treat other cancers including non-small cell lung cancers (NSCLC) expressing PD-L1. We present a case of a 66-year-old male who was admitted to the hospital for generalized gastrointestinal complaints consisting of abdominal pain, nausea, vomiting, and loose stools occurring five times daily. Labs were significant for acute transaminitis with hyperbilirubinemia and elevated lipase. All workup for infectious and noninfectious causes was negative. The patient was started pembrolizumab 15 days before admission and his previous labs had all been normal before the initiation of treatment. He was treated with high dose of steroids with initial improvement and discharged on oral steroids taper. He subsequently presented again with worsening liver function tests (LFTs) results and was restarted on a higher dose but unable to tolerate it due to steroid-induced psychosis and left the hospital against medical advice. Presented the third time with persistent elevation of LFTs and worsening hyperbilirubinemia, this time he was started on different class of steroid with atypical antipsychotic but left again a few days later seeking care at a tertiary care institute. This case highlights one of the severe side effects of the immune checkpoint inhibitors which is acute hepatitis that can sometimes lead to acute liver failure. Prompt treatment with steroids is indicated for these patients; and those who are refractory or intolerant to steroids can be treated with a multimodal approach using topical steroids, N-acetylcysteine (NAC), ursodeoxycholic acid and immune suppressant drugs.
\end{abstract}

Keywords: PD-L1; Pembrolizumab-induced hepatitis; Pancreatitis; Non-small cell lung cancer

Manuscript submitted July 16, 2018, accepted August 13, 2018

${ }^{a}$ Department of Medicine, St. Mary Medical Center, Long Beach, CA, USA bepartment of Hematology/Oncology, St. Mary Medical Center, Long Beach, CA, USA

'Pacific Shores Medical Group, Long Beach, CA, USA

${ }^{\mathrm{d} C o r r e s p o n d i n g ~ A u t h o r: ~ M u h a m m a d ~ M u f t i, ~ S t, ~ M a r y ~ M e d i c a l ~ C e n t e r, ~ G M E, ~}$ 1050 Linden Ave, Long Beach, CA, 90813, USA.

Email: muneebmufti71@gmail.com

doi: https://doi.org/10.14740/jmc3131w

\section{Introduction}

Immune checkpoint inhibitors are a novel approach to treat cancers. First used for the treatment of malignant melanomas with promising results, they were later expanded to treat other cancers including non-small cell lung cancer (NSCLC) expressing PD-L1. One of the severe side effects of this class of drug is acute hepatitis, which is a diagnosis of exclusion. Here we will present a case of a patient who was in relatively good health, recently diagnosed with NSCLC and started on pembrolizumab and presented to the hospital with acute hepatitis and pancreatitis.

\section{Case Report}

A 66-year-old male was admitted to the hospital for generalized gastrointestinal complaints consisting of abdominal pain, nausea, vomiting, and loose stools occurring five times daily. The abdominal pain was epigastric in location, sudden in onset, intermittent, worsened with food, relieved with bowel rest, 10/10 in severity; and he has never had this constellation of symptoms before. His medical history was significant for hypertension, hyperlipidemia, chronic kidney disease, HIV complicated by neuropathy and highly active antiretroviral treatment (HAART)-associated lipodystrophy, and recently diagnosed stage IV NSCLC. Of note, immunohistochemistry revealed the malignancy to have high expression of PD-L1; and he was given his first infusion of pembrolizumab 15 days before admission. Labs prior to infusion revealed both normal liver and pancreatic function.

Labs on admission were notable for a 5 -fold increase in his liver function panel, 10-fold increase in lipase and GGT, yet normal coagulation levels and ammonia level; and both acetaminophen and alcohol were undetectable (Table 1). Viral hepatitis panel was also checked and found to be negative. Home medications at that time included dolutegravir, emtricitabine, tenofovir, gabapentin, pantoprazole, folic acid, cyanocobala$\mathrm{min}$, and he had previously been on benazepril and atorvastatin, but these had been stopped 1 year prior. The patient underwent an abdominal ultrasound that showed a distended gallbladder with suggesting of gallbladder sludge, normal thickness of the gallbladder wall and common bile duct. Subsequent computed tomography (CT) of the abdomen and pelvis with oral con- 
Table 1. Pertinent Labs of the Patient During Hospitalizations

\begin{tabular}{ll}
\hline Lipase (IU/L) & $906 / 588 / 746$ \\
ANA & Negative \\
ANCA & Negative \\
F-actin Ab & Negative \\
IgG4 & Normal \\
U-Tox & Amphetamine cocaine opiates \\
PTT (s) & 34.2 \\
PT (s) & $13 / 13.2 / 14.3$ \\
INR & $1.17 / 1.16 / 1.26$ \\
Acetaminophen level (\#xb5;g/mL) & 1 \\
ETOH level (mg/dL) & 10 \\
Hepatitis panel & Negative \\
Lactate (mg/dL) & 1.17 \\
CD4 count & 485 \\
Viral load & 20 \\
GGT (mg/dL) & 650 \\
Ammonia (\#xb5;mol/L) & 33 \\
Stool culture & No growth \\
\hline
\end{tabular}

trast only exhibited nonspecific perinephric stranding without evidence of bowel obstruction. Given the current workup was negative, hepatitis, pancreatitis and likely colitis were thought to be attributed as a side effect of immunotherapy.

The patient was started on methylprednisolone $100 \mathrm{mg}$ IV Q12H on hospital day 1 (post pembrolizumab day 16), with the improvement of liver function tests (LFTs) over the proceeding days and was discharged on prednisone $60 \mathrm{mg}$ daily taper. He was readmitted again (Table 1, Figs. 1, 2) 6 days later (post pembrolizumab day 23) with similar complaints and worsening total bilirubin of $8.2 \mathrm{mg} / \mathrm{dL}$, direct bilirubin $6.0 \mathrm{mg} / \mathrm{dL}$, lipase IU/L 588 IU/L, with the remainder of his LFTs still elevated yet had not normalized. He was again started on methylprednisolone at a slightly higher dose of $125 \mathrm{mg}$ IV Q12H. Laboratory panel was checked for ANA, ANCA, anti-smooth muscle antibody, and IgG4, all of which were negative. The hospital course was complicated, in that, the patient developed steroid-induced psychosis and left the hospital against medical advice the day following admission. He was again admitted 1 week later, now post pembrolizumab day 31, the highest LFTs recorded; aspartate aminotransferase (AST) $159 \mathrm{IU} / \mathrm{L}$, alanine aminotransferase (ALT) $179 \mathrm{IU} / \mathrm{L}$, total bilirubin $14.0 \mathrm{mg} / \mathrm{dL}$, direct bilirubin $10.0 \mathrm{mg} / \mathrm{dL}$, lipase $746 \mathrm{IU} / \mathrm{L}$. Given the history of steroid-induced psychosis with methylprednisolone, he was started on hydrocortisone $200 \mathrm{mg}$ IV Q8H in addition to olanzapine $5 \mathrm{mg}$ PO twice daily. Given worsening LFTs and intolerance to steroids, the plan was to start mycophenolate. But the patient decided to leave against medical advice again as he desired to be at a tertiary care hospital for his ongoing treatment. The patient presented approximately 1 month later and by that time he had gotten a percutaneous biliary drainage put in by outside hospital. He could not provide a complete history of what happened in the other hospital and he signed out against medical advice the third time before the records could be obtained.

\section{Discussion}

This case highlights a serious potential complication with the use of PD-L1 inhibitors. This type of hepatitis with an autoimmune picture in patients treated with these drugs can appear in about 1 to $5 \%$ of the patient within the first 6 to 12 weeks of initiation of treatment [1]. The diagnosis of hepatitis caused by the drug is a diagnosis of exclusion. The more common causes must be ruled out firstly which include toxic (alcohol, salicylate, acetaminophen), metabolic (nonalcoholic fatty liver disease) and infections (viral hepatitis A, B, C, etc.). The gold standard of diagnosis would be a liver biopsy; but in most of the patient with suspected drug-induced hepatitis, it is not warranted. Studies done by Johncilla et al and Kim et al on patients taking immune checkpoint inhibitor (ipilimumab) had found the histologic features of pan lobular hepatitis with CD8 positive infiltrate similar to autoimmune hepatitis [2-3].

Different approaches to the treatment of PD-L1 inhibitors have been quoted in the literature. Treatment modalities include stopping the medication, serial monitoring of the LFTs and initiation of steroids. For the patient refractory to steroids,

\section{LFT TRENDS DURING HOSPITALIZATION}

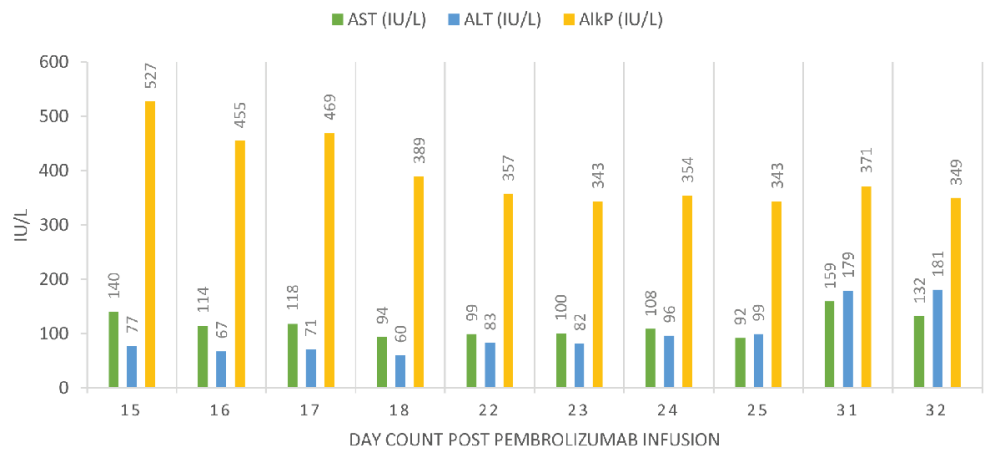

Figure 1. Liver function trends during hospitalization in relation of pembrolizumab infusion. 


\section{BILIRUBIN TRENDS DURING HOSPITALIZATION}

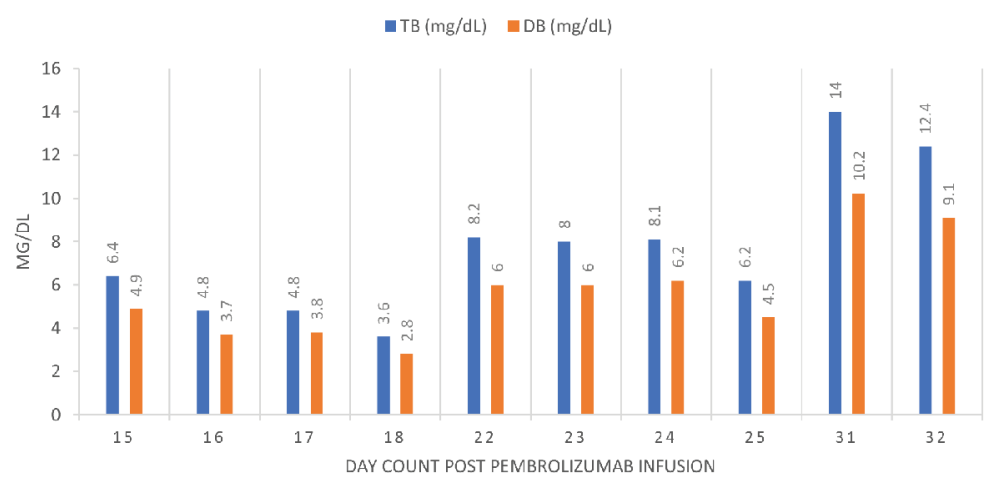

Figure 2. Liver function trends during hospitalization in relation of pembrolizumab infusion.

immune suppressant can be tried. Linardou et al suggests for hepatotoxicity related to PD-L1 inhibitors, immediate stopping of the drug and starting high dose of steroids $120 \mathrm{mg}$ IV daily as single or divided doses [4]. If no improvement is seen in 5 to 7 days duration addition of immunosuppressant like tacrolimus can be considered [4]. Mirjana Ziemer et al in the letter to the editor to Journal of Hepatology described the management of two patients with acute hepatitis secondary to nivolumab and pembrolizumab. Their approach included using initial dose methylprednisolone $1 \mathrm{mg} / \mathrm{kg}$ of body weight and taper of steroids afterward [5]. They also used topical budesonide $3 \mathrm{mg}$ three times a day with N-acetylcysteine (NAC) 1,200 mg and used ursodeoxycholic acid twice a day $500 \mathrm{mg}$ [5]. Using this approach, they were able to have LFTs normalized in 3.5 weeks\#x2019; time, and the PD-L1 inhibitors could be restarted again.

Our patient was a challenging case to treat. At first, the patient was treated with steroids with improvement and discharged home with a taper. He presented again with worsening of LFTs and was started on a higher dose of steroids but subsequently developed steroid-induced psychosis. He left the hospital against medical advice on the second admission but presented again for the third time 1 week later with worsening symptoms and further elevation of bilirubin levels. Due to his previous adverse reaction of methylprednisolone, he was started on hydrocortisone with atypical antipsychotic olanzapine. But the patient wanted to be at a tertiary care institute and left against medical advice the second time 2 days after the third admission. Even though our patient did not have biopsy-proven autoimmune hepatitis, all other workup including imaging with CT scan was negative, and the management of the patient would not have changed. On each admission, the patient\#x2019;s LFTs showed improvement with treatment, but he could not tolerate tapering of the dose or higher dose of steroids. The patient also had elevated lipase levels suggestive of acute pancreatitis which slowly down trended in his subsequent admissions but never normalized. The patient had been on HAART for HIV which can cause pancreatitis, but he had been on stable regiment for years without any abnormalities in his labs.

\section{Conclusions}

This case highlights that the immune checkpoint inhibitors like pembrolizumab and nivolumab can lead to acute hepatitis. Hospitalists should be aware of this potential side effect in the cancer patients presenting with severe transaminitis and hyperbilirubinemia. Prompt treatment with steroids or using the multimodal approach of steroids and other agents can be used to normalize liver function. Immune suppressants can be used in refractory patients.

\section{References}

1. Kahler KC, Hassel JC, Heinzerling L, Loquai C, Mossner R, Ugurel S, Zimmer L, et al. Management of side effects of immune checkpoint blockade by anti-CTLA-4 and antiPD-1 antibodies in metastatic melanoma. J\#x2005;Dtsch Dermatol Ges. 2016;14(7):662-681.

2. Johncilla M, Misdraji J, Pratt DS, Agoston AT, Lauwers GY, Srivastava A, Doyle LA. Ipilimumab-associated hepatitis: clinicopathologic characterization in a series of 11 cases. Am\#x2005;J\#x2005;Surg Pathol. 2015;39(8):1075-1084.

3. Kim KW, Ramaiya NH, Krajewski KM, Jagannathan JP, Tirumani SH, Srivastava A, Ibrahim N. Ipilimumab associated hepatitis: imaging and clinicopathologic findings. Invest New Drugs. 2013;31(4):1071-1077.

4. Linardou H, Gogas H. Toxicity management of immunotherapy for patients with metastatic melanoma. Ann Transl Med. 2016;4(14):272.

5. Ziemer M, Koukoulioti E, Beyer S, Simon JC, Berg T. Managing immune checkpoint-inhibitor-induced severe autoimmune-like hepatitis by liver-directed topical steroids. J\#x2005;Hepatol. 2017;66(3):657-659. 